\title{
Pay for Performance in der stationären Versorgung - Probleme und Lösungen
}

J. Malzahn

Qualitätsmanagement

Schlüsselwörter

Qualitätssicherung

Qualitätsmanagement

Routinedaten

Pay for Performance (P4P)

Keywords

pay for performance

quality management

administrative data

Institut

AOK-Bundesverband, Berlin

Bibliografie

DOI $10.1055 / s-0029-1242684$

Dtsch Med Wochenschr 2009;

134: S330 • @ Georg Thieme

Verlag KG Stuttgart · New York .

ISSN 0012-0472

Korrespondenz

Jürgen Malzahn

AOK-Bundesverband

Rosenthaler Str. 31

10178 Berlin

Tel. 030/34646 2491

eMail juergen.malzahn@

bv.aok.de
Pay for Performance (P4P) ist in Deutschland angekommen und alle - Ärzte, Krankenhäuser, Kassen und Politiker - sind euphorisch, was die Einsatzmöglichkeiten in der Vergütung von medizinischen Leistungen betrifft. Wenn man es nicht mit dem deutschen Gesundheitswesen und damit dem größten Einzelmarkt der gesamten Volkswirtschaft zu tun hätte, fiele es leicht die positive Grundstimmung zu teilen. Da es aber um die Verteilung erheblicher Finanzmittel geht, ist eine kritische Haltung nicht fehl am Platze. Es ist zu erwarten, dass Politik, Kassen und Leistungserbringer sehr unterschiedliche Konzepte von P4P zugrunde legen.

Im Vortrag wird anhand eines fiktiven Beispiels darauf eingegangen wie P4P konkret in die Leistungsvergütung integriert werden kann. Dabei wird gezeigt, dass bei kalkulierten oder verhandelten Preisen P4P als ergänzendes Vergütungselement gelten könnte. Weiterhin wird ein Ansatz zur Feststellung von guter Qualität auf Basis von Routinedaten vorgestellt. Dazu werden Qualitätsindikatoren definiert, die sich auf die indikationsspezifischen dem Krankenhausaufenthalt nachgelagerten Leistungen beziehen. Es wird dabei davon ausgegangen, dass gute Qualität sich durch unterdurchschnittliche Inanspruchnahme des Gesundheitswesens in den definierten Indikatoren abbildet. Außerdem wird der Frage nachgegangen, welche Konsequenzen in einem P4P-Modell ein Leistungserbringer erfahren soll, der dauerhaft die definierten Qualitätsziele nicht erreicht. Da Abschläge weder der Kasse noch den Patienten etwas nutzen, muss in einem rechtssicheren Verfahren der (partielle) Ausschluss von der Versorgung entsprechender Leistungserbringer erwirkt werden. Abschließend wird die Notwendigkeit gesetzlichen Anpassungsbedarfs beschrieben, der einen Einsatz dieses Modells außerhalb von Selektiv-Verträgen ermöglichen würde.

Autorenerklärung: Der Autor erklärt, dass keine relevanten finanziellen Verbindungen in Bezug auf dieses Manuskript bestehen. 